\title{
Development of a multiscale solver with sphere partitioning tracking
}

\author{
C. Peña-Monferrer ${ }^{1}$, J. L. Muñoz-Cobo ${ }^{1}$, G. Monrós-Andreu ${ }^{2} \&$ \\ S. Chiva ${ }^{2}$ \\ ${ }^{1}$ Institute for Energy Engineering, Universitat Politècnica de València, \\ Spain \\ ${ }^{2}$ Department of Mechanical Engineering and Construction, \\ Universitat Jaumé I, Castello de la Plana, Spain
}

\begin{abstract}
A new method to compute the dispersed phase in a Lagrangian framework is shown in this contribution for computing incompressible bubbly flows. Each bubble is divided dynamically in equivolumetric elements and tracked into the Eulerian mesh for an appropriate assignment of the effect of the bubble in the cell. The coupling between phases is done considering in the momentum equation the interfacial forces along the bubble path during an Eulerian time step. The bouncing of the bubbles between themselves and the wall is modeled with a dynamic soft sphere model. The computational results obtained for different flow conditions are validated with the recently released experimental data on upward pipe flow. The test section used is a $52 \mathrm{~mm}$ pipe of $5500 \mathrm{~mm}$ of length maintained under adiabatic conditions with air and water circulating fluids. Time-averaged results of radial distribution for void fraction, chord length, number of bubbles detected, turbulence kinetic energy, dispersed and continuous velocity profiles show a good agreement. Keywords: CFD, two-phase flow, bubbly flow, vertical pipe flow, Lagrangian, sphere partitioning, Soft-sphere collisions, OpenFOAM ${ }^{\circledR}$.
\end{abstract}

\section{Introduction}

Two-phase flow is present in natural and industrial processes of different nature as chemical and nuclear reactors, oil flow or heat exchangers. In these activities can be required to know the exact flow behavior to enhance productivity, efficiency or for ensuring the safety of plant design and production activity such as in nuclear 
industry. However, the understanding of two-phase flow is far from the level of the single-phase flow knowledge.

Due to its importance and challenges related to predict the two-phase flow structure using computational methods, two-phase flow have been investigated over the years being a research focus with significant strides accomplished to date. Computational simulations employing very different approaches were used to predict the two-phase flow behavior at different levels [1,2]. The choice of a specific method applied to industrial applications depends on its suitability for the range of operating conditions, the scale and the complexity of the domain to investigate. In this contribution a new discrete particle method using a sphere partitioning tracking of each bubble is presented with the aim of compute a large number of bubbles without resolving the interface.

The Eulerian-Lagrangian (EL) approach started some decades ago for the mathematical simulation of sprays, O'Rourke [3,4] developed a new approach coupling the Lagrangian equation for droplet distribution function developed in [5] with an Eulerian description. In their calculations, velocity and pressure were obtained by means of the Navier-Stokes equations while the motion of each particle were solved using the Newton's second law as well as the particle-particle, particle-wall, coalescence and breakup during the particle path. Lately, Dukowicz [6] developed a EL two-way coupling including momentum coupling and volume effects for computational particles representing group of particles with the same characteristics. The reader is referred to [7] for details about LE methods. For the most part, this method has been applied to sprays or particle-laden flows. Further and in a lesser extent, EL were applied to bubbly flows applications [8-10]. In this works bubble columns were simulated.

Alternatively, experimental work has been done to analyze the two-phase flow behavior and to assess the validity of existing and novel computational methods. Firstly, by focusing on the objective of the experiment, the early researches were carried out to analyze the motion of single bubbles and its effect on the liquid, already since 1900 as in [11] and later contributions [11-15]. Experiments in channels [16, 17] and bubble columns [18-22] represented the most popular research topic during decades. Two-phase pipe flow in horizontal, vertical or inclined angle has also traditionally been investigated [23,24].

A new solver has been developed and implemented in the framework of the open source package OpenFOAM ${ }^{\circledR}$ based on the PISO algorithm coupled with the Lagrangian equation of motion. The problem of effectively distribute the source term and void fraction in the grid cells for resolved bubbles is usually addressed with template functions as in [25]. However for arbitrary unstructured grids this assignment can give unrealistic or non-accurate results. Instead, in this contribution the sphere is divided in several points inside each bubble and tracked with the center of mass.

We focus the attention on polydisperse bubbly flow applications in vertical pipes. Simulations with this simple geometry let us to test the models under wellknown boundary conditions and its validity is extensible to a large number of 
industrial applications. In this paper the solver is validated to compute a $5.5 \mathrm{~m}$ section of pipe flow with the results of [26].

\section{Governing equations}

The motion of each single bubble is computed by solving the equation of motion in a Lagrangian frame:

$$
m_{b} \frac{d U_{b}}{d t}=\sum_{b=1}^{n} F_{b} .
$$

The total force $F_{b}$ acting on a single bubble is given by the sum of various forces modeled as a function of flow parameters:

$$
F_{\mathrm{b}}=F_{\text {buoyancy }}+F_{\text {virtual mass }}+F_{\text {drag }}+F_{\text {lift }}+F_{\text {wall lubrication }}+F_{\text {collisions }} \text {, }
$$

with the drag modeled using the empirical correlations for a fluid sphere of Laín et al. [27], virtual mass as Drew and Lahey [28], lift as Tomiyama et al. [29] and wall lubrication force as Antal et al. [30].

The collisions producing the bubble-bubble and bubble-wall bouncing are modeled as a spring mass. In this approach the elastic behavior of the bubbles colliding is modeled as a Hookean spring as described in fig. 1. The stiffness of the spring $K_{b_{i}}(\mathrm{~N} / \mathrm{m})$ in each single bubble $\left(b_{i}\right)$ with radius $R_{i}, a_{i}$ and $b_{i}$ the semiminor axis and semi-major axis respectively, is determined as a function of the increase of the surface area due to the bubble deformation. Assuming that the deformation of the bubble conserves the volume, the surface energy increase due to surface deformation of the spherical bubble into a oblate spheroid:

$$
\Delta E=\sigma\left(S_{\text {oblate }_{b_{i}}}-S_{\text {sphere }_{b_{i}}}\right)=\frac{1}{2} K_{b_{i}}\left(R_{b_{i}}-a_{b_{i}}\right)^{2}
$$

The value of $K_{b_{i}}$ can be obtained from Eq. 3, however, the stiffness of the bubble is not constant but acts as a nonlinear spring. In Sato et al. [31] is shown that $K_{b_{i}}$ depends on the degree of the deformation (or aspect ratio $\chi$ ) but not on the size of the bubble, resulting in

$$
K_{b_{i}}=0.85 \chi^{0.6}-0.13
$$

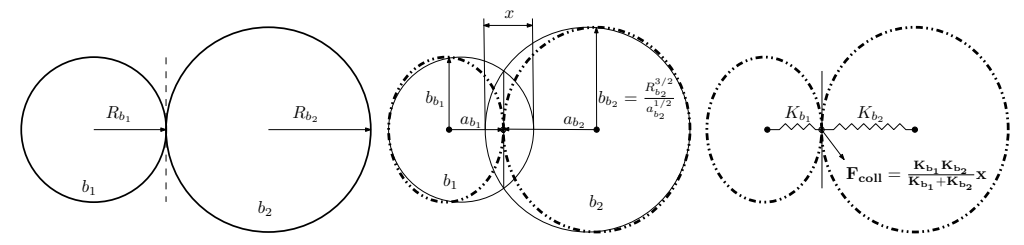

Figure 1: Spring scheme in bubble deformation. 
On the other hand, the instantaneous liquid velocity $\left(u_{1}\right)$ used to compute the forces is computed as the sum of the mean liquid velocity $U_{1}$ and the fluctuating velocity component $u_{1}^{\prime}(t)$. As previously done by several researchers as in [32], $u_{1}^{\prime}(t)$ is predicted with a discrete random walk (DRW) model [33]. The mean liquid velocity is solved with the Navier-Stokes liquid-phase momentum conservation equation with the Lagrangian coupled to include the liquid volume fraction $\left(\alpha_{1}=\right.$ $1-\alpha_{\mathrm{g}}$ ) and the momentum source contribution, $M_{\mathrm{d}}$, generated by each bubbles along its path on the cell. $M_{\mathrm{d}}$, obtained from the motion equation, represents the force from the disperse phase on the fluid phase per unit mass of fluid:

$$
\frac{\partial}{\partial t} \alpha_{1} U_{1}+\nabla \cdot\left(\alpha_{1} U_{1} U_{1}\right)=-\alpha \nabla p_{r g h}-\alpha(g \cdot x)+\nabla \cdot \tau+M_{\mathrm{d}}
$$

The turbulence is modeled with the two-phase extension of the single-phase standard $\kappa$ - $\varepsilon$ turbulence model [34]. The two-phase wall law for adiabatic bubbly boundary layer of [35] is applied.

The bubbles are injected in the system assuming a normal distribution with sizes known from the experiments. The bubbles rising in the pipe expand because of the pressure changes. The bubble size and volume variation are calculated based on the gas and liquid pressure differences and the ideal gas law as in Muñoz-Cobo et al. [36] and applied each time the bubbles moves.

\section{Code implementation}

The solver is based on the PISO algorithm coupled with a generic Lagrangian tracking integrated with the Verlet Leapfrog algorithm. A variation of the algorithm shown in $[37,38]$ is implemented in OpenFOAM ${ }^{\circledR}$ to track the bubbles along the cells. The motion of bubbles is done for unstructured, arbitrary polyhedral meshes leading with 3D meshes of complex geometries including the tracking in a parallel decomposed case.

Instead of tracking each bubble as a point-like particle we have developed an algorithm to track a fixed number of representative points inside the bubble. The distribution of this points is done strategically with a equivolumetric partitioning of the sphere with the algorithm of [39]. Each sphere (or bubble in this application) is divided in $2 n^{3}$ number of elements, depending on the number of " $n$ " shells specified for each bubble. The bubbles are divided dynamically as a function of its radius $\mathrm{R}$, computing $n=R / d r$ and applying a ceiling function (next largest integer) to specify the number of shells. According to the Eulerian mesh, a maximum value of $d r$ is established. Figure 2 shows an example for a bubble of $2.5 \mathrm{~mm}$ radius divided by 3 shells.

The residence time of each element in the cells is computed to determine the contribution of momentum and volume fraction into the grid. 


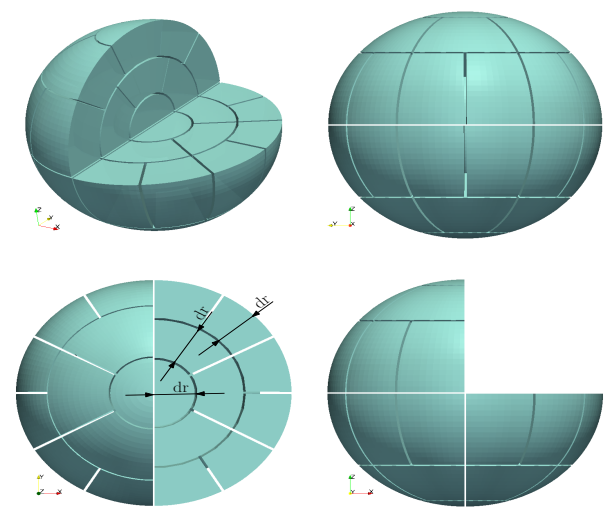

Figure 2: Bubble partition view.

\section{Description of the experimental facility}

The experimental facility is located at Universitat Jaume I, Spain. Experiments have been carried out by using an upward flow experimental loop with inner diameter, D, of $52 \mathrm{~mm}$ and $5500 \mathrm{~m}$ of length, z. Operating fluids were purified water $(\sim 30 \mu \mathrm{S} / \mathrm{m})$ and air, mixed at bottom of the section in a mixing chamber through 4 sparger (mean porosity $\sim 40 \mu \mathrm{m}$ ).

Three axial locations are used for the measurements: $\mathrm{z} / \mathrm{D}=22.4, \mathrm{z} / \mathrm{D}=61.0$ and $\mathrm{z} / \mathrm{D}=98.7$. Four-sensor conductivity probes and Laser Doppler Anemometry (LDA) techniques are adopted to extract information from the air-water flow field (fig. 3a). The measurement system consisted of three mounted four-sensor conductivity probes, mechanical traversers, a measurement circuit, a digital highspeed acquisition board, and the software used to signal processing. The foursensor conductivity probe was attached to the mechanical traverser mounted on a specially designed flange, and it could be moved along the radial direction of the test section using controlled step motors. The measurement circuit was used to measure the potential difference between the exposed tip and the grounded terminal. A high-speed NI SCXI-1000 acquisition board and a PC were used to acquire the voltage signal of the four-sensor probe, with the help of a control program developed under NI LabView software environment. More details can be found in Monrós-Andreu et al. [26].

\section{Dispersed phase variables calculation: experimental vs CFD data}

To obtain the flow characteristics of the dispersed phase is not trivial. The wellestablished conductivity probes system used in the experiments has been adapted to the CFD code. 


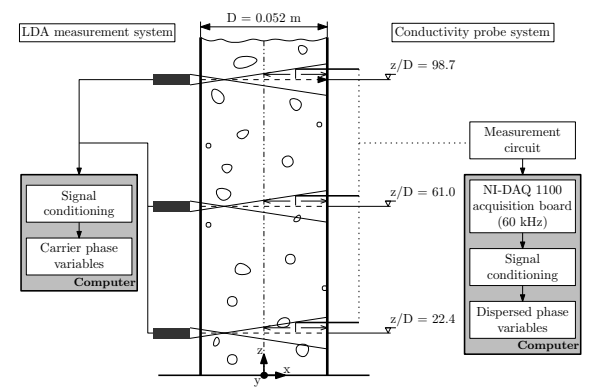

(a) LDA and conductivity probe system

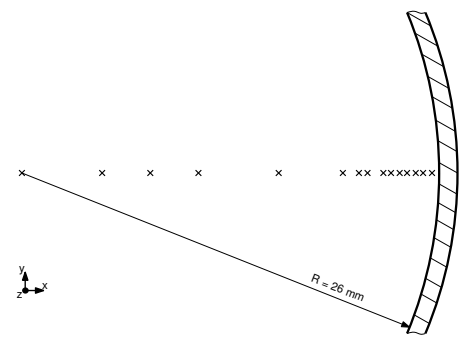

(b) Conductivity probe locations

Figure 3: Experimental configuration to obtain the two-phase flow variables.

Conductivity probes basically act as a phase identifier. Applied to the experiments, the probe is connected to a power supply with a fixed voltage, due to the large difference in conductivity between the liquid phase and the gas phase, the impedance signal acquire vary depending on phase surrounding tips. When the tip is surrounded by liquid, a lower voltage is put out; and when the tip contacts with gas, a higher voltage is obtained. But due to the finite size of each sensor and the time delay needed to wet or rewet the sensor tips, the output signal of the foursensor probe differs from ideal two-state square-wave and the signal is processed to obtain the regenerated square-wave signals.

In the simulation some probe-points are situated at the same place as in the experiments (fig. 3b). The time-averaged values of void fraction, velocity, chord length and number of bubbles detected are obtained at the probes.

\section{CFD setup}

In order to validate the solver, a pipe of $1.955 \mathrm{~m}$ of length corresponding to the distance between two measurement sections $(\mathrm{z} / \mathrm{D}=61.0$ and $\mathrm{z} / \mathrm{D}=98.7)$ is modeled and simulated. The mesh was created with the native OpenFOAM ${ }^{\circledR}$ mesh generation tool blockMesh. After the mesh sensitivity analysis was carried out, a mesh of 140,800 elements with around $4 \mathrm{~mm}$ of axial distance between nodes and 10 radial nodes. Water and air properties are calculated with usual correlations function of the operating temperature assuming tap water.

\section{Results}

Two different flow conditions at ambient temperature are used to validate the solver. The parameters defining the conditions of the experiments are described in table 1. The CFD results presented were run in a $2 x$ 6-core Intel Xeon E5645 at $2.40 \mathrm{GHz}$ with a computational costs of around 50 hours on a single processor for a physical run time of 10 seconds. 
Table 1: Flow conditions.

\begin{tabular}{lcccc}
\hline Label & $\left(j_{\mathrm{c}}\right)_{z=0}(\mathrm{~m} / \mathrm{s})$ & $\left(j_{\mathrm{d}}\right)_{z=0}(\mathrm{~m} / \mathrm{s})$ & $\left\langle\alpha_{\mathrm{d}}\right\rangle_{z / D=61}(-)$ & $T_{\mathrm{c}}\left({ }^{\circ} \mathrm{C}\right)$ \\
\hline JL05JG005 & 0.5 & 0.05 & $6.62 \times 10^{-3}$ & 20.63 \\
JL10JG005 & 1.0 & 0.05 & $4.34 \times 10^{-3}$ & 19.80 \\
\hline
\end{tabular}

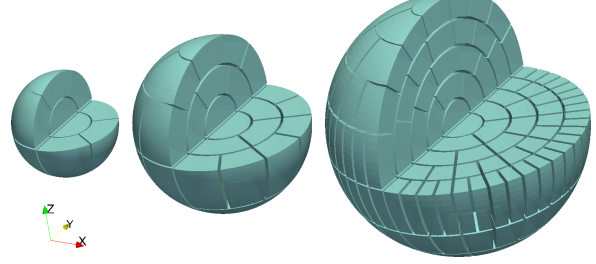

Figure 4: View of the bubbles with minimum, mean and maximum diameters considering the $95 \%$ of the sizes of the normal distribution obtained experimentally.

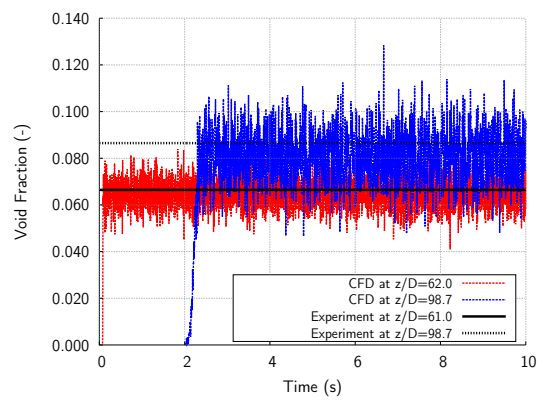

(a) JL05JG005

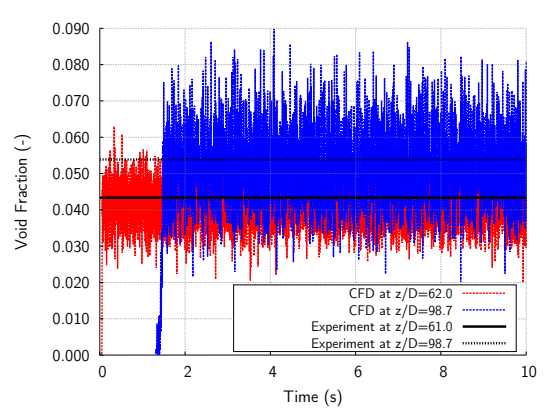

(b) JL10JG005

Figure 5: Cross-section average evolution of void fraction at two different planes.

The solver is assessed by comparing the flow characteristics with radial profiles at $\mathrm{z} / \mathrm{D}=98.7$.

The partition method described in Section 3 results in the bubble distribution shown in fig. 4 for the minimum, mean and maximum bubble injected diameters for the JL10JG005 scenario. The 95\% of bubble sizes assuming a normal distribution are injected. Under this flow condition close to 10,000 bubbles are present and tracked in the pipe with this method each time step.

The time evolution of the cross-section average void fraction at inlet $(\mathrm{z} / \mathrm{D}=$ $61.0)$ and outlet $(\mathrm{z} / \mathrm{D}=98.7)$ of the simulation is shown in fig. 5 and compared 


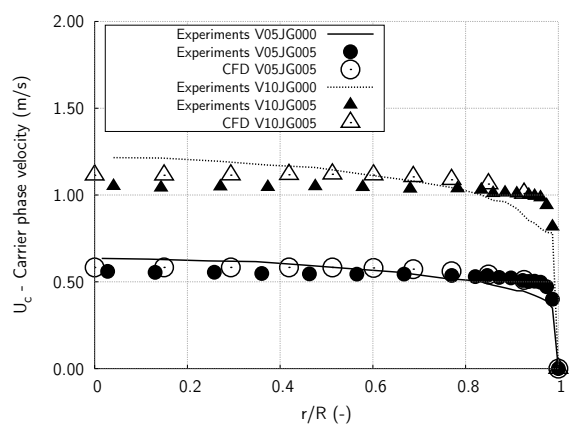

(a) Continuous phase velocity

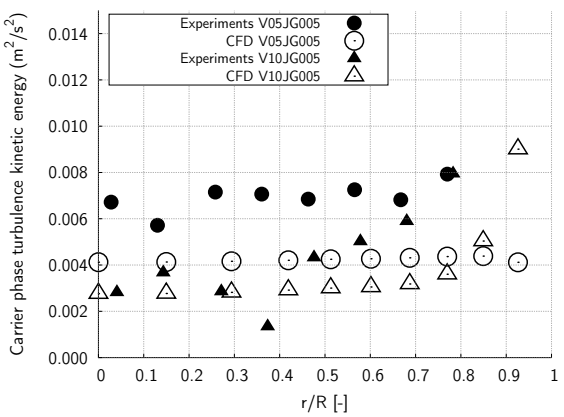

(b) Continuous phase turbulence kinetic energy

Figure 6: CFD continuous phase comparison with experimental data.

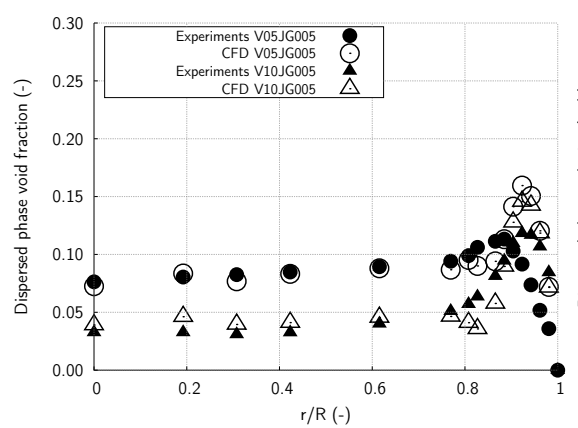

(a) Void fraction

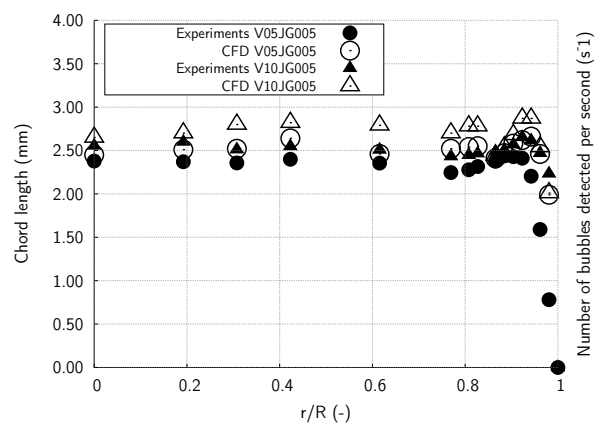

(c) Chord lenght

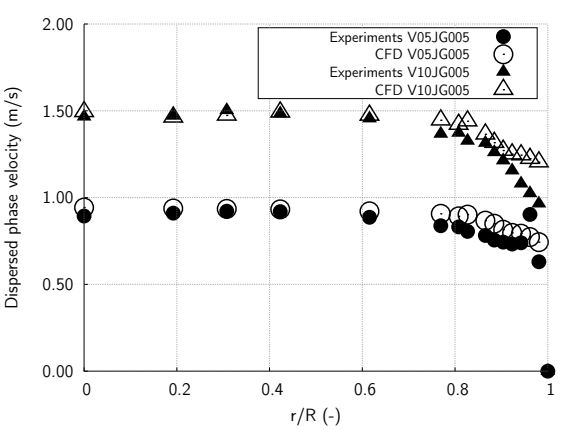

(b) Dispersed phase velocity

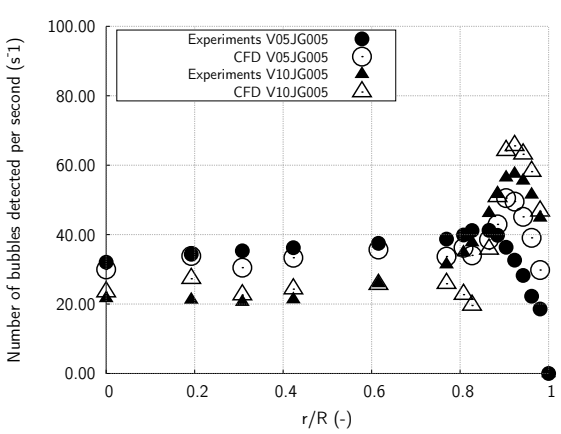

(d) Number of bubbles detected

Figure 7: CFD dispersed phase comparison with experimental data.

with the time-averaged experimental data. The void fraction at the inlet match the experimental data, which is useful to check that the injected air mass flow rate at the inlet is correct. The bubble expansion along the pipe is properly predicted as shown with the time evolution of the void fraction at the outlet of the simulation. 
In addition, this indicates from when the averaging to obtain the results could start.

The hydrodynamics of the liquid phase are evaluated with velocity and turbulence kinetic energy profiles in fig. 6 . In addition the experimental velocity profiles without gas are included to highlight the influence of the bubbles on the carrier velocity flattening the profile. The computational results of velocity are in good agreement with the experiments. The mean kinetic energy profile of the turbulence was quantified experimentally by mean of the velocity fluctuations as $\kappa_{1}=1 / 2\left(\left\langle u_{x}^{\prime 2}\right\rangle+\left\langle u_{y}^{\prime 2}\right\rangle+\left\langle u_{z}^{\prime 2}\right\rangle\right)$. It is worth noticing that within the narrow distance from the wall of around $5 \mathrm{~mm}$, the LDA techniques can capture hardly these quantities. The results for the turbulence show a similar trend and are reasonably well predicted.

The procedure described in Section 5 is used to obtain dispersed phase void fraction, velocity, chord length and number of bubbles detected. In fig. 7 the results of this solver are shown with an overall good agreement with the experimental results. The results are promising and further work will be done to validate the solver under a wide range of flow conditions.

\section{Conclusions}

A new solver to simulate polydisperse bubbly flows in adiabatic vertical pipes was discussed and successfully implemented into OpenFOAM ${ }^{\circledR}$.

Satisfactory agreement between experimental measurements and numerical results was obtained for the void fraction profiles, chord lengths, dispersed and continuous phase velocities, number of bubbles detected and continuous phase turbulence kinetic energy. Future work will be focused on testing the solver validity on regime transitions and in flow conditions with higher inlet dispersed phase void fraction.

\section{Acknowledgements}

The authors sincerely thank the "Plan Nacional de I+D+i" Project EXPERTISER ENE2010-21368-C02-01 and ENE2010-21368-C02-02 for funding the project.

\section{References}

[1] Ishii, M. \& Hibiki, T., Thermo-fluid Dynamics of Two-Phase Flow. Smart Energy Systems, Springer, 2006.

[2] Prosperetti, A. \& Tryggvason, G., Computational Methods for Multiphase Flow. Cambridge University Press, 2007.

[3] O'Rourke, P., Collective drop effects on vaporizing liquid sprays. Ph.D. thesis, 1981. 
[4] O'Rourke, P., The kiva computer program for multidimensional chemically reactive fluid flows with fuel sprays. Numerical Simulation of Combustion Phenomena, eds. R. Glowinski, B. Larrouturou \& R. Temam, Springer Berlin Heidelberg, volume 241 of Lecture Notes in Physics, pp. 74-89, 1985.

[5] Williams, F.A., Spray combustion and atomization. Physics of Fluids (19581988), 1(6), pp. 541-545, 1958.

[6] Dukowicz, J.K., A particle-fluid numerical model for liquid sprays. Journal of Computational Physics, 35(2), pp. 229-253, 1980.

[7] Subramaniam, S., Lagrangian-Eulerian methods for multiphase flows. Progress in Energy and Combustion Science, 39(2-3), pp. 215-245, 2013.

[8] Deflnoij, E., Lammers, F., Kuipers, J. \& van Swaaij, W., Dynamic simulation of dispersed gas-liquid two-phase flow using a discrete bubble model. Chemical Engineering Science, 52(9), pp. 1429-1458, 1997.

[9] Laín, S., Bröder, D., Sommerfeld, M. \& Göz, M., Modelling hydrodynamics and turbulence in a bubble column using the Euler-Lagrange procedure. International Journal of Multiphase Flow, 28(8), pp. 1381-1407, 2002.

[10] E. Shams, Finn, J. \& Apte, S., A Numerical Scheme for Euler-Lagrange Simulation of Bubbly Flows in Complex Systems. International Journal for Numerical Methods in Fluids, 2010.

[11] Allen, H.S., XXXI. The motion of a sphere in a viscous fluid. Philosophical Magazine Series 5, 50(304), pp. 323-338, 1900.

[12] Davies, R. \& Taylor, G., The mechanics of large bubbles rising through extended liquids and through liquids in tubes. Proceedings of the Royal Society of London Series A Mathematical and Physical Sciences, 200(1062), pp. 375-390, 1950.

[13] Peebles, F.N. \& Garber, H.J., Studies on the motion of gas bubbles in liquids. Chemical Engineering Progress, 49(2), pp. 88-97, 1953.

[14] Aybers, N. \& Tapucu, A., The motion of gas bubbles rising through stagnant liquid. Wärme - und Stoffübertragung, 2(2), pp. 118-128, 1969.

[15] Duineveld, P.C., The rise velocity and shape of bubbles in pure water at high Reynolds number. Journal of Fluid Mechanics, 292, pp. 325-332, 1995.

[16] Troniewski, L. \& Ulbrich, R., Two-phase gas-liquid flow in rectangular channels. Chemical Engineering Science, 39(4), pp. 751-765, 1984.

[17] Zenit, R., Koch, D.L. \& Sangani, A.S., Measurements of the average properties of a suspension of bubbles rising in a vertical channel. Journal of Fluid Mechanics, 429, pp. 307-342, 2001.

[18] Kumar, S.B., Computed tomographic measurements of void fraction and modeling of the flow in bubble columns. Ph.D. thesis, Florida Atlantic University Boca Raton, FL, 1994.

[19] Kumar, S., Dudukovic, M. \& Toseland, B., Chapter 1 - measurement techniques for local and global fluid dynamic quantities in two and three phase systems. Non-Invasive Monitoring of Multiphase Flows, ed. J.C.L.P. Dudukovic, Elsevier Science B.V.: Amsterdam, pp. 1-45, 1997. 
[20] Kumar, S.B., Moslemian, D. \& Duduković, M.P., Gas-holdup measurements in bubble columns using computed tomography. AIChE Journal, 43(6), pp. 1414-1425, 1997.

[21] Degaleesan, S., Fluid dynamic measurements and modeling of liquid mixing in bubble columns. Ph.D. thesis, Washington University, 1997. Department of Chemical Engineering, 1997.

[22] Chen, J., Li, F., Degaleesan, S., Gupta, P., Al-Dahhan, M.H., Dudukovic, M.P. \& Toseland, B.A., Fluid dynamic parameters in bubble columns with internals. Chemical Engineering Science, 54(13-14), pp. 2187-2197, 1999.

[23] O'brien, M.P. \& Gosline, J.E., Velocity of large bubbles in vertical tubes. Industrial \& Engineering Chemistry, 27(12), pp. 1436-1440, 1935.

[24] Chiva, S., Julia, J.E., Hernandez, L., Mendez, S., Muñoz-Cobo, J.L. \& Romero, A., Experimental study on two-phase flow characteristics using conductivity probes and laser doppler anemometry in a vertical pipe. Chem Eng Commun, 197(2), pp. 180-191, 2009.

[25] Deen, N., van Sint Annaland, M. \& Kuipers, J., Multi-scale modeling of dispersed gas-liquid two-phase flow. Chemical Engineering Science, 59(89), pp. 1853-1861, 2004. Complex Systems and Multi-scale Methodology.

[26] Monrós-Andreu, G., S. Chiva, R. Martínez-Cuenca, S. Torró, J. E. Juliá, L. Hernández \& R. Mondragón, Water temperature effect on upward airwater flow in a vertical pipe: Local measurements database using four-sensor conductivity probes and LDA. EPJ Web Conf, 45, p. 01105, 2013.

[27] Laín, S., Bröder, D. \& Sommerfeld, M., Experimental and numerical studies of the hydrodynamics in a bubble column. Chemical Engineering Science, 54(21), pp. 4913-4920, 1999.

[28] Drew, D.A. \& Lahey, R.T., The virtual mass and lift force on a sphere in rotating inviscid flow. Int J Multiphase Flow, 13(1), pp. 113-121, 1987.

[29] Tomiyama, A., Tamai, H., Zun, I. \& Hosokawa, S., Transverse migration of single bubbles in simple shear flows. Chemical Engineering Science, 57(11), pp. 1849-1858, 2002.

[30] Antal, S., Lahey Jr, R.T. \& Flaherty, J., Analysis of phase distribution in fully developed laminar bubbly two-phase flow. International Journal of Multiphase Flow, 17(5), pp. 635-652, 1991.

[31] Sato, A., Shirota, M., Sanada, T. \& Watanabe, M., Modeling of bouncing of a single clean bubble on a free surface. Physics of Fluids, 23(1), p. 013307, 2011.

[32] Buwa, V.V., Deo, D.S. \& Ranade, V.V., Eulerian-Lagrangian simulations of unsteady gas-liquid flows in bubble columns. International Journal of Multiphase Flow, 32(7), pp. 864-885, 2006.

[33] Gosman, A. \& Loannides, E., Aspects of computer simulation of liquidfueled combustors. Journal of Energy, 7(6), pp. 482-490, 1983.

[34] Launder, B. \& Spalding, D., The numerical computation of turbulent flows. Comput Method Appl M, 3(2), pp. 269-289, 1974. 
[35] Troshko, A. \& Hassan, Y., A two-equation turbulence model of turbulent bubbly flows. International Journal of Multiphase Flow, 27(11), pp. 19652000, 2001.

[36] Muñoz-Cobo, J., Chiva, S., Essa, M. \& Mendes, S., Experiments performed with bubbly flow in vertical pipes at different flow conditions covering the transition region: simulation by coupling Eulerian, Lagrangian and 3D random walks models. Archives of Thermodynamics, 33(1), pp. 3-39, 2012.

[37] Vaidya, A.M., Subbarao, P.M.V. \& Gaur, R.R., A novel and efficient method for particle locating and advancing over deforming, non-orthogonal mesh. Numerical Heat Transfer, Part B: Fundamentals, 49(1), pp. 67-88, 2006.

[38] Macpherson, G.B., Nordin, N. \& Weller, H.G., Particle tracking in unstructured, arbitrary polyhedral meshes for use in CFD and molecular dynamics. Communications in Numerical Methods in Engineering, 25, p. 273, 2008.

[39] Yang, G. \& Chen, I.M., Equivolumetric partition of solid spheres with applications to orientation workspace analysis of robot manipulators. IEEE Transactions on Robotics, 22(5), pp. 869-879, 2006. 